\title{
LOCALIZATION OF AGE-DEPENDENT ANTI-CROWDING POPULATIONS
}

\author{
BY \\ GASTÓN E. HERNÁNDEZ \\ University of Connecticut, Storrs, Connecticut
} \begin{abstract}
age-dependence and diffusion

$$
\begin{aligned}
\frac{\partial \rho}{\partial t}+\frac{\partial \rho}{\partial a} & =\left(\rho u_{x}\right)_{x}-\mu(a, u) \rho \\
\rho(x, t, 0) & =\int_{0}^{\infty} \beta(a, u) \rho(x, t, a) d a \\
\rho(x, 0, a) & =\rho_{0}(x, a) \geq 0
\end{aligned}
$$
\end{abstract}

Abstract. In this work we prove the existence of solutions and study the localization and nonlocalization of the population in the Gurtin-MacCamy model with

where

and

$$
u(x, t)=\int_{0}^{\infty} \rho(x, t, a) d a
$$

$$
\beta(a, u)=\beta(u) \sum_{k=1}^{n} b_{k} a^{k} e^{-\alpha a}, \quad \mu(a, u)=\mu(u) .
$$

1. Introduction. We consider here a nonlinear one-dimensional population dispersal problem with age-dependence. Let $\rho(x, t, a)$ denote the number of individuals per unit age and unit length who are of age $a$ at time $t$ and position $x$. The total population at $x$ and $t$ is

$$
u(x, t)=\int_{0}^{\infty} \rho(x, t, a) d a .
$$

Assuming that the population disperses to avoid crowding, Gurtin and MacCamy [9] arrived at the following equations:

$$
\begin{aligned}
\frac{\partial \rho}{\partial t}+\frac{\partial \rho}{\partial a} & =k\left(\rho u_{x}\right)_{x}-\mu(a, u) \rho, \\
\rho(x, t, 0) & =\int_{0}^{\infty} \beta(a, u) \rho(x, t, a) d a, \\
\rho(x, 0, a) & =\rho_{0}(x, a) \geq 0
\end{aligned}
$$

where $k$ is a constant that can be normalized to be 1 .

Received July 27, 1992.

1991 Mathematics Subject Classification. Primary 92A, 35K; Secondary 35A.

Key words and phrases. Populations, diffusion, age-dependence. 
The balance law (1.2) is of Malthusian type. If age and diffusion are ignored, the population would tend to decay exponentially depending on the death modulus $\mu$. On the other hand, if only diffusion is neglected and $\mu$ is assumed to depend on $a$, the balance equation reduces to

$$
\frac{\partial \rho}{\partial t}+\frac{\partial \rho}{\partial a}=-\mu(a) \rho
$$

which can be readily integrated along characteristics.

Letting $t=a-t_{0}$ for $a \leq t$ and $t=a+a_{0}$ for $t \leq a$, a formal solution is obtained:

$$
\rho(t, a)= \begin{cases}\rho(0, a-t) e^{-\int_{0}^{t} \mu(a-t+s) d s}, & t \leq a, \\ \rho(t-a, 0) e^{-\int_{0}^{t} \mu(s) d s}, & t \geq a .\end{cases}
$$

Although $\rho(0, a-t)=\rho_{0}(a-t)$ is the prescribed initial age distribution, the term $\rho(t, 0)$ needs to be determined. Assuming that the population-sex ratio remains constant, the birth rate $\beta(a, u)$ is defined such that $\beta(a, u) d a$ represents the average number of offsprings produced per unit time by an individual aged between $a$ and $a+d a$. In this form Eq. (1.3) is the birth law and $\beta(a, u)$ is called the birth module.

The diffusion mechanism in Eq. (1.2) is such that the flux of individuals is proportional to the gradient of the total population. The first model considering diffusion was given by Skellam [22], in 1951. With $\rho$ independent of age he assumed random diffusion of individuals, which gives a balance law of the form

$$
\frac{\partial \rho}{\partial t}=k \frac{\partial^{2} \rho}{\partial x^{2}}+\sigma(t)
$$

where $k$ is constant. It has been observed, however, that several species actually disperse to avoid crowding rather than dispersing randomly (see, for instance, Carl [4] for squirrel populations and Murray [20] for insect populations). This fact is modeled by considering

$$
\frac{\partial \rho}{\partial t}=\frac{\partial^{2} \varphi}{\partial x^{2}}(\rho)+\sigma(\rho),
$$

where $\varphi^{\prime}(0)=0, \varphi^{\prime}(\rho)>0$ for $\rho>0$. This was done by Gurtin and MacCamy in [10]. In [7] Gurney and Nibset arrived at a similar equation with $\varphi(\rho)=\rho^{2}$ after considering a probabilistic walk in which individuals either stay at their present location or move in a direction of decreasing population.

The system (1.1)-(1.4) is just too general to be treated in that form and some simplifying assumptions are necessary. In [11] Gurtin and MacCamy assumed that $\mu(a, u)=\mu_{0}$ and $\beta(a, u)=\beta_{0} e^{-\alpha a}$, where $\mu_{0}, \beta_{0}$, and $\alpha$ are constants, which reduce the system to a pair of partial differential equations. The first supposition models the case of a harsh environment in which age is not a significant cause of death (for instance a population in the presence of predators that do not discriminate with age), the latter corresponds to a population with a higher fertility at age 0 . Of course, such an assumption is not to be taken literally but rather as an approximation 
to higher fertility at younger ages. In [14] and [16] the author studied the existence of a solution and the spatial localization of the population when

$$
\beta(a, u)=\beta_{0}(u) e^{-\alpha a}
$$

and

$$
\mu(a, u)=\mu_{0}(u),
$$

where $\beta_{0}, \mu_{0}$ are smooth positive functions. This problem was proposed in [8] by M. Gurtin.

A more realistic birth modulus was suggested in [12] and [11], namely,

$$
\beta(a, u)=\beta(u) a e^{-\alpha a},
$$

with an expected number of zero births at age $a=0$, an increasing fertility up to a maximum age $a=\frac{1}{\alpha}$, and then a monotonic decrease to zero. Murray [20] suggests that for human-like populations the birth modulus looks like $\beta(a, u)=\beta(u) g(a)$, where

$$
g(a)= \begin{cases}0, & 0 \leq a \leq a_{0}, \\ \left(a-a_{0}\right) e^{-\alpha a}, & a \geq a_{0} .\end{cases}
$$

In [8] M. Gurtin proposed a very general birth function:

$$
\beta(a, u)=\beta(u) \sum_{k=1}^{n} b_{k} a^{k} e^{-\alpha a}
$$

which would reduce Eqs. (1.1)-(1.4) to a mixed system of $n+1$ nonlinear partial differential equations.

It is also possible to consider

$$
\beta(a, u)=\beta(u) \sum_{k=0}^{n} b_{k} g_{k}(a),
$$

where $S=\left\{g_{1}(a), g_{2}(a), \ldots, g_{n}(a)\right\}$ is a set of nonnegative continuously differentiable functions on $[0, \infty)$ that is closed under differentiation, i.e.,

$$
g_{i}^{\prime}(a)=\sum_{k=1}^{n} c_{i k} g_{k}(a), \quad i=1,2, \ldots, n .
$$

$S=\left\{e^{-\alpha a}\right\}$ would correspond to the case in [14], while $S=\left\{e^{-\alpha a}, a e^{-\alpha a}\right\}$ is proposed in [11].

In this paper we consider $S=\left\{e^{-\alpha a}, a e^{-\alpha a}, \ldots, a^{n} e^{-\alpha a}\right\}$ that corresponds to Eq. (1.13) with $g_{k}(a)=a^{k-1} e^{-\alpha a}, k=1, \ldots, n$. Without loss of generality, we assume the $b_{k}^{\prime} \mathrm{s}$ to satisfy $\sum_{k=0}^{n} b_{k} g_{k}(a) \leq 1$ on $[0, \infty)$.

As an example, note that the birth function (1.12), for human-like populations, is very well approximated by $g(a)=50(a(a-0.5)(a-1))^{4} e^{-5 a}$, where $a$ is in 10-year units. See Fig. 1 on p. 38.

The model discussed here appears to contain most of the essential aspects of a general birth function and is still mathematically treatable. 


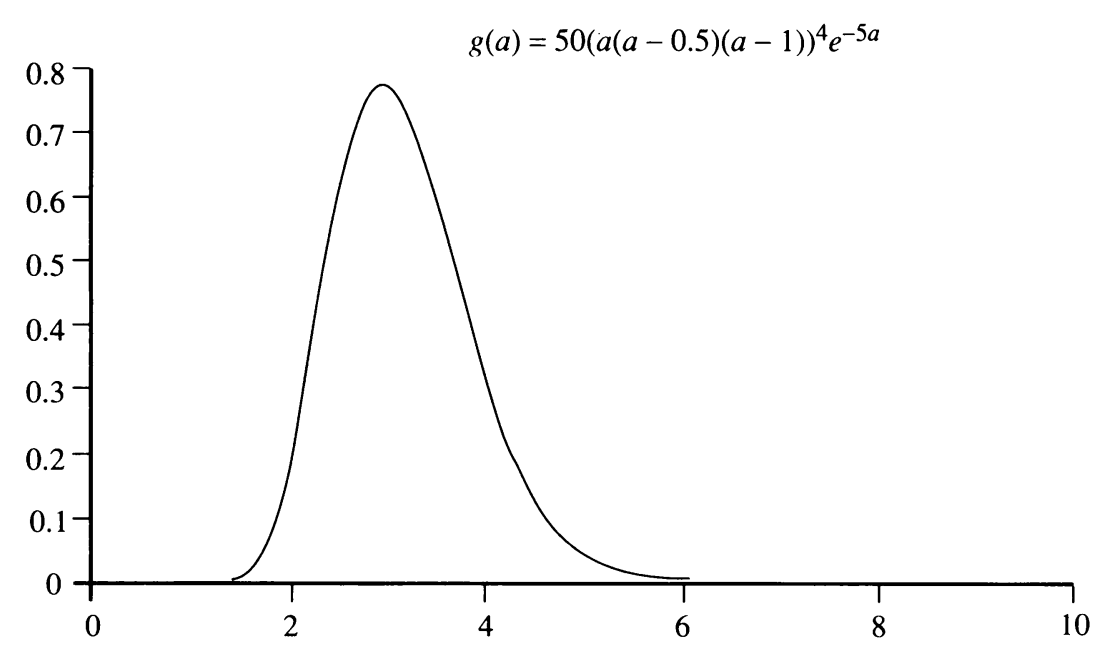

FIG. 1.

Thus in this work we consider $\beta(a, u)$ as in Eq. (1.13) and $\mu(a, u)$ as in Eq. (1.10). For the model obtained with these $\beta, \mu$ we prove the existence of solutions, the existence of populated and unpopulated regions, and the localization of the population. Our scheme is as follows:

In Sec. 2 using Eq. (1.13) we reduce the problem to a system of $n+1$ partial differential equations and provide an appropriate definition of weak solutions for this system. In Sec. 3 we prove the existence of solutions by applying a fixed-point theorem to $(\varepsilon, n)$-approximating systems and then showing the convergence when $n \rightarrow \infty$ and $\varepsilon \rightarrow 0$. In Sec. 4 , by comparing the solution $u$ to the solutions of the Porous Medium equation, we show that the $\mathbb{R} \times[0, \infty)$ domain will be divided into two regions: the populated region in which $u>0$ and the unpopulated region in which $u=0$. Finally in Sec. 5 we give conditions in $\beta$ and $\mu$ under which the population will remain localized in a fixed region or will eventually cover the whole space.

Results for the existence of solutions and the localization of the populations in higher dimensions have been obtained in [17] when the birth modulus is given by Eq. (1.9) and the initial distribution is radially symmetric.

From another point, Busenberg and Ianelli [3] proved the existence of solutions when both the birth and death modules are independent of total population and depend only on age, $\mu(a, u)=\mu_{0}(a), \beta(a, u)=\beta_{0}(a)$. The latter supposition renders the birth law (1.3) linear in $u$.

Gurtin and MacCamy [13] looked into product solutions of the form $\rho(x, t, a)=$ $g(a) u(x, t)$, under the assumption that $\beta(a, u)=g(a)$ (linear birth law), and the death process is the form

$$
\mu(a, u)=\mu_{\mathrm{n}}(a)+\mu_{\mathrm{e}}(u)
$$

with $\mu_{\mathrm{n}}(a)$ the probability of dying of natural causes during $(a, a+d a)$ and $\mu_{\mathrm{e}}(u)$ the probability of death due to environmental factors during the same interval.

For the random dispersal model we refer the reader to Garroni-Langlais [6], Langlais [19], DiBlassio [5], and the references contained therein. 
2. Weak solutions. We introduce auxiliary functions

$$
G_{i}(x, t)=\int_{0}^{\infty} g_{i}(a) \rho(x, t, a) d a, \quad i=1, \ldots, n .
$$

The birth law can then be written as

$$
\rho(x, t, 0)=\beta(u) \sum_{i=1}^{n} b_{i} G_{i}(x, t) .
$$

Integrating Eq. (1.2) with respect to $a$ from 0 to $\infty$ and assuming $\rho=0$ at $a=\infty$ we obtain for $i=1,2, \ldots, n$

$$
\frac{\partial u}{\partial t}=\frac{\partial}{\partial x}\left(u \frac{\partial u}{\partial x}\right)-\mu(u) u+\beta(u) \sum_{j=1}^{n} b_{j} G_{j}(x, t) ;
$$

multiplying Eq. (1.2) by $g_{i}(a)$ and integrating we obtain

$$
\begin{aligned}
\frac{\partial G_{i}}{\partial t}= & \frac{\partial}{\partial x}\left(G_{i} \frac{\partial u}{\partial x}\right)-\mu(u) G_{i} \\
& +\sum_{j=1}^{n}\left(g_{i}(0) \beta(u) b_{j}+c_{i j}\right) G_{j}(x, t) .
\end{aligned}
$$

Note that if $\rho(x, t, a)>0$ in a set of positive measure, then $G_{i}(x, t)>0$. On the other hand, if $\rho(x, t, a)=0$ a.e. in $a$, then $G_{i}(x, t)=u(x, t)=0$. Thus we can define the per capita birth rate for $u=0, i=1,2, \ldots, n$,

$$
q_{i}(x, t)=\frac{G_{i}(x, t)}{u(x, t)} \text { for } u \neq 0,
$$

and $q_{i}(x, t) \equiv 0$ for $u=0$.

Substituting $G_{i}=u q_{i}$ into Eqs. (2.2) and (2.3) and including the corresponding initial conditions we arrive at

$$
\begin{gathered}
\frac{\partial u}{\partial t}=\frac{\partial}{\partial x}\left(u \frac{\partial u}{\partial x}\right)+\left(\beta(u) \sum_{j=1}^{n} b_{j} q_{j}-\mu(u)\right) u \\
\frac{\partial q_{i}}{\partial t}-\frac{\partial u}{\partial x} \frac{\partial q_{i}}{\partial x}=\sum_{j=1}^{n}\left(\beta(u) b_{j}\left(d_{i}-q_{i}\right)+c_{i j}\right) q_{j}, \quad i=1,2, \ldots, n ; \\
u(x, 0)=u_{0}(x)=\int_{0}^{\infty} \rho_{0}(x, a) d a \geq 0 ; \\
q_{i}(x, 0)=q_{i 0}(x)=\frac{\int_{0}^{\infty} g_{i}(a) \rho(x, t, a) d a}{\int_{0}^{\infty} \rho(x, t, a) d a}
\end{gathered}
$$

where $d_{i}=g_{i}(0)$.

These are the equations corresponding to any basis set $S$. For the particular basis set considered here, we also have $d_{1}=1, d_{i}=0, i=1,2, \ldots, n$, and for $i, j=1, \ldots, n, c_{i i}=-\alpha, c_{i+1, i}=i$, and $c_{i j}=0$ otherwise. 
Let

$$
z(x, t)=\sum_{j=1}^{n} b_{j} q_{j}(x, t)
$$

The previous system is

$$
\begin{aligned}
\frac{\partial u}{\partial t} & =\frac{\partial}{\partial x}\left(u \frac{\partial u}{\partial x}\right)+(\beta(u) z-\mu(u)) u \\
\frac{\partial q_{1}}{\partial t}-\frac{\partial u}{\partial x} \frac{\partial q_{1}}{\partial x} & =-\alpha q_{1}-\beta(u) q_{1} z+\beta(u) z \\
\frac{\partial q_{i}}{\partial t}-\frac{\partial u}{\partial x} \frac{\partial q_{i}}{\partial x} & =-\alpha q_{i}+-\beta(u) q_{i} z+(i-1) q_{i-1}, \quad i=2,3, \ldots, n \\
u(x, 0) & =u_{0}(x), \quad q_{i}(x, 0)=q_{i 0}(x), \quad i=1, \ldots, n
\end{aligned}
$$

Equations (2.9)-(2.12) form a mixed parabolic-hyperbolic system of $n+1$ differential equations.

Let $h(x, t, u)=\beta(u) z(x, t)-\mu(u)$. When $h \equiv 0$, Eq. (2.9) is the porous medium equation

$$
\frac{\partial u}{\partial t}=\frac{\partial}{\partial x}\left(u \frac{\partial u}{\partial x}\right), \quad u(x, 0)=u_{0}(x) \geq 0 .
$$

It models the diffusion of a homogeneous gas flow through a homogeneous porous medium. There is a large literature for this equation. (Complete references are given in Aronson [1], Peletier [21], and Vazquez [23].)

The most striking difference between the solutions of Eq. (2.13) and those of the usual heat equation

$$
\frac{\partial u}{\partial t}=\frac{\partial^{2} u}{\partial x^{2}}, \quad u(x, 0)=u_{0}(x)
$$

is their speed of propagation. Assume a population that satisfies Eq. (2.14) is initially distributed in a bounded interval $I_{0}$, i.e., $u_{0}(x)>0$ in $I_{0}, u_{0}(x) \equiv 0$ on $\mathbb{R}-I_{0}$. The solutions of Eq. (2.14) are characterized by an infinite speed of propagation: $u(x, t)>0$ for all $x \in \mathbb{R}, t>0$. The population would spread immediately to all the space. On the other hand, if $u$ satisfies Eq. (2.13) it will have a finite speed of propagation. If $I_{0}=\left(x_{1}, x_{2}\right)$, there are two monotone interface curves $\gamma_{1}(t), \gamma_{2}(t)$ with $x_{1}=\gamma_{1}(0), x_{2}=\gamma_{2}(0)$ that separate the region of positivity of $u$ from the region in which $u \equiv 0$.

In this work we shall assume that the initial population is nonnegative and satisfies $u_{0}(x)>0$ on $I_{0}, u_{0}(x) \equiv 0$ on $\mathbb{R}-I_{0}$. It is reasonable to expect then a behavior similar to the solutions of the porous medium equation.

In [16] it is proved that when $\beta(a, u)=\beta(u) e^{-\alpha a}$, the support of $u(x, t)$ increases with $t$ and is always on the interval. Further, if

$$
\sup _{0 \leq u \leq M_{1}} \frac{\mu(u)}{\beta(u)}<\inf _{0 \leq u \leq M_{1}} \frac{\beta(u)-\alpha}{\beta(u)},
$$

then the support of $u(x, t)$ grows to $(-\infty, \infty)$ as $t \rightarrow \infty$. In this case all the real line will be ultimately populated. On the other hand, if

$$
\sup _{0 \leq u \leq M_{1}} \frac{\beta(u)-\alpha}{\beta(u)}<\inf _{0 \leq u \leq M_{1}} \frac{\mu(u)}{\beta(u)},
$$


then the population remains localized in an interval $[-L, L]$ for all times. In this case the interaction between age-dependence and diffusion produces that the population persists in a limited region. Similar behavior is described in [17] for the problem with radial symmetry in several variables. In this work we discuss in detail similar localization results when $n=2$, i.e., $\beta(a, u)=\beta(u) a e^{-\alpha a}$.

It is well known that Eq. (2.13) does not have classical solutions unless the initial datum is strictly positive. (See Aronson [2] and Kalashnikov [18].) This is because if $u_{0}$ has compact support, the solutions will not have a continuous first derivative when crossing the interfaces. A fortiori, Eq. (2.9) will not have the classical solution either.

We shall consider the following definition of weak solution:

Let $K=\left\{\varphi(x, t) \in C^{\infty}\left(\Omega_{T}\right) / u \equiv 0\right.$ for large $|x|$ and near $\left.t=T\right\}$.

Given $\varphi \in K$, multiply Eq. (2.9) by $\varphi$ and integrate on $\mathbb{R} \times(0, T)$ to obtain

$$
\begin{aligned}
\int_{0}^{T} \int_{\mathbf{R}} & \left(\frac{1}{2} \frac{\partial\left(u^{2}\right)}{\partial x} \frac{\partial \varphi}{\partial x}-u \frac{\partial \varphi}{\partial t}\right) d x d t \\
= & \int_{0}^{T} \int_{\mathbf{R}}\left(\sum_{j=1}^{n} \beta(u) b_{j} q_{j}-\mu(u)\right) u \varphi d x d t \\
& +\int_{\mathbf{R}} u_{0}(x) \varphi(x, 0) d x .
\end{aligned}
$$

Next multiplying Eq. (2.6) by $q_{j}$ and Eq. (2.17) by $u$, adding, and integrating we obtain

$$
\begin{aligned}
\int_{0}^{T} \int_{\mathbf{R}} & \left(\frac{1}{2} q_{i} \frac{\partial\left(u^{2}\right)}{\partial x} \frac{\partial \varphi}{\partial x}-u q_{i} \frac{\partial \varphi}{\partial t}\right) d x d t \\
= & \int_{0}^{T} \int_{\mathbf{R}}\left(\sum_{j=1}^{n}\left(\beta(u) b_{j}+c_{i j}\right) q_{j} u-\mu(u) q_{i} u\right) d x d t \\
& +\int_{\mathbf{R}} u_{0}(x)\left(q_{i}\right)_{0}(x) \varphi(x, 0) d x .
\end{aligned}
$$

Define a weak solution of Eqs. (2.9)-(2.12) as an $(n+1)$-vector $\left(u, q_{1}, \ldots, q_{n}\right)^{\mathrm{T}}$ such that $\frac{\partial u}{\partial x} \in L_{\mathrm{loc}}^{2}\left(\Omega_{T}\right)$ in the sense of distributions, $q_{i}(x, t) \in L_{\mathrm{loc}}^{2}\left(\Omega_{T}\right), i=$ $1, \ldots, n$, and Eqs. (2.17), (2.18) are satisfied for any $\varphi \in K$.

The following notation will be used: $C^{2,1}\left(\Omega_{T}\right)$ is the Banach space consisting of functions $u(x, t)$ defined in $\Omega_{T}$ with continuous second derivatives in $x$ and continuous first derivative in $t . C^{\alpha}\left(\Omega_{T}\right)$ is the Banach space of functions $u(x, t)$ defined in $\Omega_{T}$ for which the $\alpha$-norm

$$
\|u\|_{\alpha}=\sup _{\Omega_{T}}|u|+\sup _{\Omega} \frac{|u(x, t)-u(y, s)|}{|x-y|^{\alpha}+|t-s|^{\alpha / 2}}
$$

is bounded. $C^{1+\alpha}\left(\Omega_{T}\right)$ is the Banach space for which the $(1+\alpha)$-norm $\|u\|_{1+\alpha}=$ $\left\|\frac{\partial u}{\partial x}\right\|_{\alpha}+\left\|\frac{\partial u}{\partial t}\right\|_{\alpha}+\|u\|_{\alpha}$ is bounded. Similarly, $C^{2+\alpha}\left(\Omega_{T}\right)$ is the Banach space for which the $(2+\alpha)$-norm $\|u\|_{2+\alpha}=\left\|\frac{\partial^{2} u}{\partial x^{2}}\right\|_{\alpha}+\|u\|_{1+\alpha}$ is bounded. 
3. Existence results. Concerning the functions $\mu(u), \beta(u)$, and $\rho_{0}(x, a)$ the following assumptions will be adopted throughout.

- $\beta(r), \mu(r)$ are bounded continuously differentiable functions on $[0, \infty)$.

- $\rho_{0}(x, a)$ is sufficiently smooth so that $u_{0}$ and $q_{0 i}, i=1, \ldots, n$, are bounded continuous functions in $\mathbb{R}$, and $u_{0}(x)$ is continuously differentiable.

The continuous differentiability assumption on $\beta$ and $\mu$ as well as $u_{0}$ can be relaxed to require only continuity. We have preferred to avoid this kind of generality in order to keep the notation simple. For definiteness it is assumed that there exist constants $M_{0}$ and $K_{0}$ such that $u_{0},\left|u_{0}^{\prime}\right| \leq M_{0}$ and $\beta, \mu,\left|\beta^{\prime}\right|,\left|\mu^{\prime}\right| \leq K_{0}$.

THEOREM 3.1. Under the previous assumptions there exists a (weak) solution $\left(u, q_{1}, \ldots, q_{n}\right)^{\mathrm{T}}$ of Eqs. (2.6)-(2.9). The population $u$ is uniformly bounded in $\mathbb{R}$, and $u^{2}$ is differentiable with respect to $x$.

Theorem 3.2. Assume there exist $x_{1}, x_{2} \in \mathbb{R}$ such that $\rho_{0}(x, a)>0$ for all $x \in$ $I_{0}=\left(x_{1}, x_{2}\right)$ and $\rho_{0}(x, a)=0$ for $x \in \mathbb{R} \backslash I_{0}$. Then the support of $u(x, t)$ is a finite interval for every $t$, and there exist two interface curves $\gamma_{1}(t), \gamma_{2}(t)$ such that $x_{1}=\gamma_{1}(0), x_{2}=\gamma_{2}(0)$, and $\operatorname{supp} u(\cdot, t)=\left[\gamma_{1}(t), \gamma_{2}(t)\right]$ for every $t$.

In Sec. 4 we study the localization of $u$.

Proof of Theorem 3.1. Let

$$
\mathbf{q}=\left(q_{1}, q_{2}, \ldots, q_{n}\right)^{\mathrm{T}}, \quad C=\left(c_{i j}\right)_{i, j=1, \ldots, n}, \quad A(\mathbf{q})=\left(a_{i j}\right),
$$

where for $i, j=1, \ldots, n$

$$
a_{1 j}(\mathbf{q})=b_{j}\left(1-q_{1}\right), \quad a_{i j}(\mathbf{q})=-b_{j} q_{i} .
$$

Then Eqs. (2.10)-(2.12) can be written as the nonlinear vector equation

$$
\begin{aligned}
\frac{\partial \mathbf{q}}{\partial t}-\frac{\partial u}{\partial x} \frac{\partial \mathbf{q}}{\partial x} & =(\beta(u) A(\mathbf{q})+C) \mathbf{q}, \\
\mathbf{q}_{0}(x, 0) & =\mathbf{q}_{0}(x)=\left(q_{01}(x), \ldots, q_{0 m}(x)\right)^{\mathrm{T}} .
\end{aligned}
$$

We shall first study the existence of a solution of this system for a given $u$ and strictly positive initial data.

Lemma 3.1. If $u \in C^{2+\alpha}\left(\Omega_{T}\right)$ is given, $u \geq 0$, there exists a unique solution $\mathbf{q} \in$ $C^{1+\alpha}\left(\Omega_{T}\right)$ of the system

$$
\begin{aligned}
\frac{\partial \mathbf{q}}{\partial t}-\frac{\partial u}{\partial x} \frac{\partial \mathbf{q}}{\partial x} & =(\beta(u) A(\mathbf{q})+C) \mathbf{q}, \\
\mathbf{q}(x, 0) & =\mathbf{q}_{0}(x) .
\end{aligned}
$$

Proof. Define the characteristics $x(t ; \bar{x}, \bar{t})$ by

$$
\begin{gathered}
\frac{\partial x}{\partial t}=-\frac{\partial u}{\partial x}(x, t), \\
x(\bar{t} ; \bar{x}, \bar{t})=\bar{x} .
\end{gathered}
$$


Since $\frac{\partial u}{\partial x}$ is Lipschitz continuous, there is always a local solution of this equation. Since $\frac{\partial u}{\partial x}$ is also bounded, this solution can be made global by extending it to the boundary of $[0, T] \times \mathbb{R}$.

The function $\mathbf{p}(t)=\mathbf{q}(x(t ; \bar{x}, \bar{t}), t)$ satisfies

$$
\begin{aligned}
\frac{d \mathbf{p}}{d t} & =(\beta(u) A(\mathbf{p})+C) \mathbf{p}, \\
p(0) & =q_{0}(x(0)) .
\end{aligned}
$$

The right-hand side is a Lipschitz continuous function; so a solution $\mathbf{p}(t)$ exists for small $t$ and $\mathbf{p}$ nonnegative. We shall obtain a priori bounds for $\mathbf{p}$ that will ensure the existence of global solutions of Eqs. (3.7).

For $i=1, \ldots, n$, Eqs. (2.6) are

$$
\begin{aligned}
& \frac{d p_{1}}{d t}=-\alpha p_{1}+\beta(u) z-\beta(u) p_{1} z, \\
& \frac{d p_{2}}{d t}=-\beta(u) p_{2} z+p_{1}-\alpha p_{2}, \\
& \frac{d p_{3}}{d t}=-\beta(u) p_{3} z+2 p_{2}-\alpha p_{3}, \\
& \quad \vdots \\
& \frac{d p_{n}}{d t}=-\beta(u) p_{n} z+(n-1) p_{n-1}-\alpha p_{n} .
\end{aligned}
$$

Let $w=1-p_{1}$; the first equation becomes

$$
\frac{d w}{d t}+(\beta(u) z) w=\alpha p_{1} .
$$

From Eq. (2.8) and $g_{1}(a)=e^{-\alpha a}$ it follows that $p_{1}(0)<1$; so $w(0)>0$ and $w(t)>0$ for all $t$. Thus, $0 \leq p_{1}(t) \leq 1$ for all $t$. For $m \geq 1$, assume $p_{m}$ is nonnegative and bounded by a constant $k_{m}$. Then

$$
\frac{d p_{m+1}}{d t} \leq-\alpha p_{m}+m k_{m}
$$

so $p_{m+1}(t) \leq \max \left\{\frac{m k_{m}}{\alpha}, p_{m+1}(0)\right\}$. Since $p_{1}(t)$ is bounded by 1 , it follows by the previous argument that all $p_{m}^{\prime} \mathrm{s}$ are nonnegative and uniformly bounded for all $t$. In particular, there exists a constant $K_{1}$ such that $z(t) \leq K_{1}$ for all $t \in[0, \infty)$. Thus the solution of Eqs. (3.7) exists for all $t$.

Now define

$$
\mathbf{q}(\bar{x}, \bar{t})=\mathbf{q}(x(0 ; \bar{x}, \bar{t}), 0)+\int_{0}^{\bar{t}}(\beta(u) A(\mathbf{p})+C) \mathbf{p}(t) d t .
$$

Differentiating Eqs. (3.5)-(3.6) with respect to the parameters $\bar{x}, \bar{t}$ it is shown that $x_{\bar{t}}(t)-u_{\bar{x}}(\bar{x}, \bar{t}) x_{\bar{x}}(t) \equiv 0$ for all $t$. This fact plus direct differentiation of Eq. (3.9) give that $\mathbf{q}$ is the required solution of Eqs. (3.3)-(3.4).

Let $y=(x, t)$. In $\mathbb{R}^{2}$ consider the mollifier $J(y)$ defined by

$$
J(y)= \begin{cases}k e^{-1 /\left(1-|y|^{2}\right)}, & |y|<1, \\ 0, & |y| \geq 1,\end{cases}
$$

where $k$ is a constant such that $\int_{\mathbb{R}^{2}} J(y) d y=1$. 
Let $J_{m}(y)=\frac{1}{m^{2}} J(m y)$ and $\left(q * J_{m}\right)(y)=\int_{\left|y-y^{\prime}\right| \leq 1 / m} J\left(m\left(y-y^{\prime}\right)\right) q\left(y^{\prime}\right) d y^{\prime}$.

Then if $q \in \mathscr{L}^{2},\left\{q * J_{m}\right\}_{m=1}^{\infty}$ is a $\mathscr{C}^{\infty}$-sequence that converges to $q$ in $\mathscr{L}^{2}$; if $q$ is continuous, $\left\{q * J_{m}\right\}_{m=1}^{\infty}$ converges to $q$ uniformly on compact sets. We will apply Schauder's Fixed Point Theorem to the following $(\varepsilon, n)$-approximating systems:

$$
\begin{aligned}
\frac{\partial u}{\partial t} & =\frac{\partial}{\partial x}\left(u \frac{\partial u}{\partial x}\right)+\left(\beta(u) z_{n}-\mu(u)\right) u ; \\
\frac{\partial \mathbf{q}}{\partial t}-\frac{\partial u}{\partial x} \frac{\partial q_{i}}{\partial x} & =(\beta(u) A(\mathbf{q})+C) \mathbf{q} ; \\
q_{i}(x, 0) & =q_{i 0}(x), \quad i=1, \ldots, n ; \\
u(x, 0) & =u_{0}(x),
\end{aligned}
$$

where $z_{m}=\sum_{i=1}^{n} b_{j}\left(q_{j} * J_{m}\right)$.

Let $K_{2}$ be a constant to be specified, and for $\alpha \in(0,1)$

$$
V=\left\{w \in C^{2+\alpha}\left(\Omega_{T}\right):\|w\|_{2+\alpha} \leq K_{2}, w \geq \varepsilon\right\} .
$$

Clearly, $V$ is closed and convex. Given $w \in V$, by the previous discussion there exists a unique solution depending on $w, \mathbf{q} \in C^{1+\alpha}\left(\Omega_{T}\right)$ of Eqs. (3.11)-(3.14).

Since $0 \leq q_{i} \leq K_{1},\left\|q_{i} * J_{n}\right\|_{\sigma} \leq 2 n K_{1}$ for any $\sigma \in(0,1)$. Since $u(x, 0) \geq \varepsilon$ is strictly positive, from the standard theory of partial differential equations we get that Eq. (3.11) has a unique solution $u \in \mathscr{C}^{2+\sigma}\left(\Omega_{T}\right)$. Further, there exists a constant $K_{3}$ (depending on $\varepsilon$ and $n$ ) such that $\|u\|_{2+\sigma} \leq K_{3}$. Choose $K_{2} \geq K_{3}$ and $\sigma>\alpha$. Then $T$ maps $V$ into $V$. Since bounded sets in $C^{2+\sigma}$ are precompact in $C^{2+\alpha}$ for $0<\alpha<\sigma<1$, we also obtain that $T$ is precompact. Standard estimates show that $T$ is continuous. Thus Schauder's Fixed Point Theorem implies the existence of a fixed point $u$ of $T$. This $u$ with the corresponding $\mathbf{q}$ form a solution of Eqs. (3.11)(3.14). Further, from the maximum principle it follows that $\varepsilon \leq u(x, t) \leq M_{1}$, where $M_{1}$ depends only on the initial bounds $M_{0}, K_{0}$. The solution found will be denoted by $u_{\varepsilon, n}$ and $\left(q_{i}\right)_{\varepsilon, n}$. After this, the proof of existence of solutions is similar to the one given in [17] and we will just sketch it here.

From [15] we quote the following result:

TheOREM 3.3. Let $u \in C^{2+\alpha}\left(\Omega_{T}\right)$ be a (classical) solution of

$$
\begin{aligned}
\frac{\partial u}{\partial t} & =\frac{\partial}{\partial x}\left(u \frac{\partial u}{\partial x}\right)+h(x, t, u)(u-\varepsilon), \\
u(x, 0) & =u_{0}(x)+\varepsilon,
\end{aligned}
$$

where $u_{0}(x) \geq 0$. Then the $\alpha$-norm of $u$ is uniformly bounded independently of $\varepsilon$ and the modulus of continuity of $h$; i.e., there exists $K_{3}>0$ depending only on $\left\|u_{0}\right\|_{\infty}$ and $\|h\|_{\infty}$ such that $\|u\|_{\alpha} \leq K_{3}$ in $\bar{\Omega}_{T}$.

Using the Arzela-Ascoli Theorem in the sequence $\left\{u_{\varepsilon, n}\right\}$ we extract a subsequence $\left\{u_{\varepsilon, n_{k}}\right\}$ that converges to an $\alpha$-Hölder continuous function $u_{\varepsilon}(x, t)$. Rename this sequence $\left\{u_{\varepsilon, k}\right\}$. 
Since $\left\|q_{\varepsilon, k}\right\|_{\alpha}$ is bounded independently of $k$ (but depending on $\varepsilon$ ), we can also extract subsequences $\left\{\left(q_{i}\right)_{\varepsilon, k_{l}}\right\}$ of $\left\{\left(q_{i}\right)_{\varepsilon, k}\right\}$ that converge uniformly in compact subsets of $\Omega_{T}$ to continuous functions $\left(q_{i}\right)_{\varepsilon}$, respectively. Again, rename all these sequences as labeled by $\varepsilon$ and $k$.

Since $u_{\varepsilon, k} \geq \varepsilon$, Eqs. (3.11) are uniformly parabolic in $k$. It follows then from the standard theory that $\left\|u_{\varepsilon, k}\right\|_{2+\alpha} \leq K_{4}$, a constant independent of $k$, and for $\alpha^{\prime}<\alpha$ there exists a subsequence $\left\{u_{\varepsilon, n_{k}}\right\}$ that converges to $u_{\varepsilon}$ in $\mathscr{C}^{2+\alpha^{\prime}}\left(\Omega_{T}\right)$. Clearly $u_{\varepsilon}$ and $\left(q_{i}\right)_{\varepsilon}$ satisfy Eqs. (2.17)-(2.18). Actually, it can be shown that $u_{\varepsilon}$ is a classical solution of Eq. (2.11) and that $\left(q_{i}\right)_{\varepsilon} \mathrm{s}$ are continuous. Again using that $\left\|u_{\varepsilon}\right\|_{\alpha} \leq K_{3}$ we can extract a subsequence $\left\{u_{\delta}\right\}$ that converges uniformly in compact sets to an $\alpha$-Hölder continuous function $u(x, t)$. As in [14] it can be shown that $u^{2}$ is differentiable, $\left\{\partial\left(u_{\delta}^{2}\right) / \partial x\right\}$ is uniformly bounded, and $\left\{\partial\left(u_{\delta}^{2}\right) / \partial x\right\}$ converges pointwise to $\partial u^{2} / \partial x$. The corresponding $\left\{\left(q_{i}\right)_{\delta}\right\}$ converge weakly to a vector integrable function $\mathbf{q}$, and Eqs. (2.17)-(2.18) are satisfied. Thus the pair $(u, \mathbf{q})$ is a weak solution of Eqs. (2.11)-(2.14).

4. Populated and unpopulated regions. We begin by studying the existence of the interfaces that separate the populated region from the unpopulated region.

The following two lemmas were first given in [16]. They are valid here with almost no changes, so we just sketch their proofs.

LemMA 4.1. If $u\left(x_{0}, t_{0}\right) \geq \eta>0$, then $u\left(x_{0}, t\right)>0$ for all $t \geq t_{0}$. Thus if a region becomes populated at time $t_{0}$, it will remain populated for all later times. In particular, the initial region $\left(x_{1}, x_{2}\right)$ remains populated for all times (but it might tend to 0 as $t \rightarrow \infty)$.

Proof. Assume first $t_{0}=0$ and $x_{1}<x_{0}<x_{2}$. Using the continuity of $u_{0}(x)$ choose $\delta>0$ small, $\delta<x_{0} / 2$, such that $\delta^{2} \leq \eta / 2$ and $u_{0}(x) \geq \eta / 2$ on $I_{\delta}=$ $\left(x_{0}-\delta, x_{0}+\delta\right)$.

For $x \in\left[x_{0}-\delta, x_{0}+\delta\right] \times(0, T]$, define $v(x, t)=e^{-k t}\left(\delta^{2}-\left(x-x_{0}\right)^{2}\right)+\frac{\varepsilon}{2}$, where $k=M_{1}+4,|h| \leq M_{1}$. An application of the Maximum Principle for the operator

$$
L[z]=\frac{\partial z}{\partial t}-\frac{\partial}{\partial x}\left(z \frac{\partial z}{\partial x}\right)+\left(M_{1}+2\right)(z-\varepsilon)
$$

gives that

$$
v(x, t) \leq u_{\varepsilon}(x, t) \text { on }\left[x_{0}-\delta, x_{0}+\delta\right] \times[0, T] .
$$

As $\varepsilon \rightarrow 0$ we obtain

$$
u\left(x_{0}, t\right) \geq e^{-\left(4+M_{1}\right) t} \delta^{2} .
$$

The case $t_{0}>0$ reduces to $t_{0}=0$ by using $w(x, t)=v\left(x, t+t_{0}\right)$.

LemMA 4.2. For every $\bar{t}>0$ there exists $R_{*}>0$ such that $u(x, \bar{t})=0$ for $|x|>R_{*}$. Thus the support of $u(x, t)$ is finite for every $t$.

Proof. Choose $R>\left|x_{1}\right|,\left|x_{2}\right|$ so that $u_{0}(x) \equiv 0$ for $|x|>R$.

Let $\sigma<1, \sigma<\frac{1}{K_{1}}$ (without loss of generality we assume that $K_{1}>1$ ). Fix $x_{0}$ such that $x_{0}-\frac{1}{\sigma} \geq R$ and $\tau=1 /\left(14 M_{1}\right)$. 
This time an application of the Maximum Principle with the operator $\mathscr{L}[z]=$ $\frac{\partial z}{\partial t}-\frac{\partial}{\partial x}\left(z \frac{\partial z}{\partial x}\right)-h(x, t, z)(z-\varepsilon)$ and the function

$$
v(x, t)=\sigma\left(\frac{2 \tau}{2 \tau-t}\right)\left(x-x_{0}\right)^{2}+\varepsilon\left(\frac{2 \tau}{2 \tau-t}\right)^{\sigma}
$$

gives that

$$
u\left(x_{0}, t\right) \leq \varepsilon\left(\frac{2 \tau}{2 \tau-t}\right)^{\sigma} \leq 2^{\sigma} \varepsilon
$$

on $B_{1}=\left[x_{0}-\frac{1}{\sigma}, x_{0}+\frac{1}{\sigma}\right] \times[0, \tau]$.

Repeating the argument after a finite number $k$ of steps we arrive at

$$
u_{\varepsilon}(x, t) \leq 2_{\varepsilon}^{k \sigma}
$$

for

$$
x \geq x_{k} \geq R+k \frac{1}{\sigma}, \quad 0 \leq t \leq \bar{t} .
$$

As $\varepsilon \rightarrow 0$ we obtain that $u(x, t) \equiv 0$ for

$$
x \geq R+k \frac{1}{\sigma}, \quad 0 \leq t \leq \bar{t} .
$$

Hence the lemma.

This last lemma implies that the support of $u(x, t)$ is finite for all $t$. On the other hand, by Lemma 3.1, once $u$ becomes positive it stays positive for all later times; hence the support of $u(\cdot, t)$ increases with $t$. In particular, if $\operatorname{supp} u_{0}(x)$ is an interval, another application of the Maximum Principle shows that $\operatorname{supp} u(x, t)$ is also an interval. Let $\operatorname{supp} u(\cdot, t)=\left[\gamma_{1}(t), \gamma_{2}(t)\right]$. Then if $\rho_{0}(x, a)>0$ for all $x \in\left(x_{1}, x_{2}\right)$ and $\rho_{0} \in\left(x_{1}, x_{2}\right)$ and $\rho_{0}(x, a)=0$ for $x \in \mathbb{R} \backslash\left(x_{1}, x_{2}\right)$, then the support of $u(x, t)$ is a finite interval for every $t$ and there exist two interface curves $\gamma_{1}(t), \gamma_{2}(t)$ such that $x_{1}=\gamma_{1}(0)$ and $x_{2}=\gamma_{2}(0)$ for every $t$. This proves Theorem 2 .

5. Localization. We now turn to the question of localization. The fact that the support of $u(\cdot, t)$ increases with $t$ leads us naturally to the question of determining the limit of $\operatorname{supp} u(\cdot, t)$ as $t \rightarrow \infty$.

The population is said to be localized if there exists $L>0$ such that $\operatorname{supp} u(\cdot, t) \subset$ $[-L, L]$ for all $t>0$.

Let $v(x, t)$ be the unique solution of

$$
\begin{aligned}
\frac{\partial v}{\partial t} & =\frac{\partial}{\partial x}\left(v \frac{\partial v}{\partial x}\right)-\delta v, \\
v(x, 0) & =v_{0}(x) \geq 0 .
\end{aligned}
$$

Introducing the change of independent and dependent variables, $t(s)=$ $-\frac{1}{\delta} \log (1-\delta s)$, for $0 \leq s<\frac{1}{\delta}$, and $w(x, s)=\frac{1}{1-\delta s} v(x, t(s))$. Equation (5.1) reduces to the standard porous medium equation

$$
\begin{aligned}
\frac{\partial w}{\partial s} & =\frac{\partial}{\partial x}\left(w \frac{\partial w}{\partial x}\right), \\
w(x, 0) & =v(x, 0)=v_{0}(x) .
\end{aligned}
$$


Thus $w(x, s)$ has finite support for every $s>0$. In particular, for $s=\frac{1}{\delta}$ there exists $L>0$ such that $\operatorname{supp} w\left(x, \frac{1}{\delta}\right) \subset[-L, L]$. It follows that $v$ is localized and $\operatorname{supp} v(\cdot, t) \subset[-L, L]$ for all $t>0$.

Our first localization result is

Theorem 5.1. Assume that $\beta(m) K_{1}<\mu(m)$ for $m \in\left[0, M_{1}\right]$. Then $u(x, t)$ is localized.

Proof. There exists $\delta>0$ such that $\beta(m) K_{1}-\mu(m) \leq-\delta<0$ for all $m \in$ $\left[0, K_{1}\right]$

Let $v_{\varepsilon}(x, t)$ be the classical solution of

$$
\begin{aligned}
\frac{\partial v}{\partial t} & =\frac{\partial}{\partial x}\left(v \frac{\partial v}{\partial x}\right)-\delta(v-\varepsilon), \\
v(x, 0) & =u_{0}(x)+\varepsilon \geq 0 .
\end{aligned}
$$

If $\mathscr{L}[z]=\frac{\partial z}{\partial t}-\frac{\partial}{\partial x}\left(z \frac{\partial z}{\partial x}\right)+\delta(z-\varepsilon)$, we have that $\mathscr{L}\left[v_{\varepsilon}\right]=0$ and

$$
\begin{aligned}
\mathscr{L}\left[u_{\varepsilon}\right] & =\left(\beta\left(u_{\varepsilon}\right) q_{\varepsilon}(x, t)-\mu\left(u_{\varepsilon}\right)+\delta\right)\left(u_{\varepsilon}-\varepsilon\right) \\
& <\left(\beta\left(u_{\varepsilon}\right) K_{1}-\mu\left(u_{\varepsilon}\right)+\delta\right)\left(u_{\varepsilon}-\varepsilon\right)<0 .
\end{aligned}
$$

Thus the Maximum Principle implies that $u_{\varepsilon}(x, t) \leq v_{\varepsilon}(x, t)$ in $\Omega_{T}$. As $\varepsilon \rightarrow 0$ we obtain that $u(x, t) \leq v(x, t)$, the solution of Eq. $(5.1)$, in $\Omega_{T}$. Since $v(x, t)$ is localized, so is $u(x, t)$. Hence the theorem.

From the structure of Eq. (2.5) it is clear that this result is also valid for any separated birth function $\beta(a, u)=\beta(u) g(a)$.

If $h(r, t, u)=\beta(u) q-\mu(u) \geq 0$, the comparison principle implies that $u(x, t) \geq$ $w(x, t)$, the solution of the Porous Medium Equation (5.2). It is known [18] that $\operatorname{supp} w(x, t) \rightarrow[0, \infty)$ as $t \rightarrow \infty$. Therefore, $\operatorname{supp} u(x, t) \rightarrow[0, \infty)$ in this case.

Thus, if the birth modulus is definitely less than the death modulus, the population will not diffuse further than a fixed interval. On the other hand, if $h(x, t, u) \geq 0$, the population will eventually cover all of $[0, \infty)$. Of course, to check the latter condition, more detailed knowledge about $u$ and $q$ is needed. Other conditions for localization and nonlocalization are discussed next. To simplify the presentation we consider only the case $\beta(a, u)=\beta(u) b_{0} a e^{-\alpha a}$, where $b_{0}=\frac{1}{\alpha e}$ is a normalization factor. This reduces Eqs. (2.5)-(2.8) to a system of three equations. Further, let $q=q_{2}$ and $p=q_{1} / q_{2} ;$ then

$$
\begin{gathered}
\frac{\partial u}{\partial t}=\frac{\partial}{\partial x}\left(u \frac{\partial u}{\partial x}\right)+(\beta(u) q-\mu(u)) u ; \\
\frac{\partial q}{\partial t}-\frac{\partial u}{\partial x} \frac{\partial q}{\partial x}=\left(b_{0} p-\alpha\right) q-\beta(u) q^{2} ; \\
\frac{\partial p}{\partial t}-\frac{\partial u}{\partial x} \frac{\partial p}{\partial x}=\beta(u)-b_{0} p^{2} ; \\
u(x, 0)=u_{0}(x), \quad q(x, 0)=q_{0}(x), \quad p(x, 0)=p_{0}(x) .
\end{gathered}
$$

Let

$$
\beta^{*}=\sup _{0 \leq r \leq K_{1}} \beta(r), \quad \mu_{*}=\inf _{0 \leq r \leq K_{1}} \mu(r),
$$

and $\beta_{*}, \mu^{*}$ be defined similarly. 
Also, let

$$
s_{1}=\sup _{0 \leq u \leq M_{1}} \frac{\sqrt{b_{0} \beta^{*}}-\alpha}{\beta(u)}, \quad i_{1}=\inf _{0 \leq u \leq M_{1}} \frac{\mu(u)}{\beta(u)}
$$

and

$$
s_{2}=\sup _{0 \leq u \leq M_{1}} \frac{\mu(u)}{\beta(u)}, \quad i_{2}=\inf _{0 \leq u \leq M_{1}} \frac{\sqrt{b_{0} \beta_{*}}-\alpha}{\beta(u)} .
$$

ThEOREM 5.2. Assume that $s_{1}<i_{1}$ and $q_{0}(x) \leq c_{1}$ for some $c_{1} \in\left(s_{i}, i_{1}\right)$. If also $b_{0} M_{0} \leq \sqrt{b_{0} \beta^{*}}$, then $u(x, t)$ is localized.

Proof. There exists $\delta>0$ such that

$$
\frac{\sqrt{b_{0} \beta^{*}}-\alpha}{\beta(u)}<c_{1}<\frac{\mu(u)-\delta}{\beta(u)}
$$

for every $u \in\left[0, M_{1}\right]$.

Now if $\kappa$ is a positive constant and $\kappa \neq R_{0}$, the unique solution of

$$
\frac{d R}{d t}=\kappa-b_{0} R^{2}, \quad R(0)=R_{0}
$$

is

$$
R(t)=\kappa_{1} \frac{e^{\sqrt{\kappa b_{0} t}}-C e^{-\sqrt{\kappa b_{0} t}}}{e^{\sqrt{\kappa b_{0} t}}+C e^{-\sqrt{\kappa b_{0} t}}}
$$

where $\kappa_{1}=\sqrt{\kappa / b_{0}}$ and $C=\left(\kappa_{1}-R_{0}\right) /\left(\kappa_{1}+R_{0}\right)$.

In fact, if $R_{0}<\kappa_{1}, R(t)$ increases from $R_{0}$ to $\kappa_{1}$; if $R_{0}>\kappa_{1}, R(t)$ decreases from $R_{0}$ to $\kappa_{1}$. When $R_{0}=\kappa_{1}, R=R_{0}$ is the constant solution. From this observation and standard comparison it follows that any solution of

$$
\frac{d P}{d t}=\beta(u)-b_{0} P^{2}
$$

is bounded above and below by the solutions of Eqs. (5.8) with $\kappa=\beta^{*}$ and $\kappa=\beta_{*}$ respectively. Thus if $e_{1}=\min \left\{\sqrt{\beta_{*} / b_{0}}, P(0)\right\}$ and $e_{2}=\max \left\{\sqrt{\beta^{*} / b_{0}}, P(0)\right\}$, then

$$
b_{1} \leq P(t) \leq b_{2}
$$

for any solution $P(t) \in C^{\prime}(0, T)$ of Eq. (5.9) and the same bounds are valid for the corresponding $p(x, t)$.

From these bounds we obtain that

$$
b_{0} p_{\varepsilon} \leq \max \left\{\sqrt{b_{0} \beta^{*}}, b_{0} M_{0}\right\} \leq \sqrt{b_{0} \beta^{*}} .
$$


Consider $\mathscr{L}[q]=\frac{\partial q}{\partial t}-\frac{\partial u}{\partial x} \frac{\partial q}{\partial x}-q\left(b_{0} p-\alpha-\beta q\right)$. The usual comparison principle is valid for this $q$ (see [17]).

We have $\mathscr{L}\left[q_{\varepsilon}\right]=0$ and

$$
-\mathscr{L}\left[c_{1}\right]=c_{1}\left(b_{0} p-\alpha-\beta(u) c_{1}\right) \leq c_{1} \beta(u)\left(\frac{\sqrt{b_{0} \beta^{*}}-\alpha}{\beta(u)}-c_{1}\right) \leq 0 .
$$

Thus $\mathscr{L}\left[c_{1}\right] \geq 0=\mathscr{L}\left[q_{\varepsilon}\right]$. Since $q_{0}(x) \leq c_{1}$, we get $q(x, t) \leq c_{1}$ in $\Omega_{T}$. Therefore, $\beta(u) q(x, t)-\mu(u)<-\delta<0$ and the argument of Lemma 5.1 applies. Thus $u(x, t)$ is localized.

TheOrem 5.3. Assume that $s_{2}<i_{2}$ and $c_{2}<q_{0}(x) \leq 1$ for some $c_{2} \in\left(s_{2}, i_{2}\right)$. If also $b_{0} M_{0} \geq \sqrt{b_{0} \beta_{*}}$, then $u(x, t)$ is nonlocalized, i.e., supp $u(\cdot, t) \rightarrow \infty$ as $t \rightarrow \infty$.

Proof. This time from the bounds for $p$ given in (5.10) we obtain

$$
b_{0} p_{\varepsilon} \geq \min \left\{\sqrt{b_{0} \beta_{*}}, b_{0} M_{0}\right\} \geq \sqrt{b_{0} \beta_{*}}
$$

and

$$
-\mathscr{L}\left[c_{1}\right]=c_{2}\left(b_{0} p-\alpha-\beta(u) c_{2}\right) \geq c_{2} \beta(u)\left(\frac{\sqrt{b_{0} \beta_{*}}-\alpha}{\beta(u)}-c_{2}\right) \geq 0 .
$$

Thus $\mathscr{L}\left[c_{2}\right] \leq 0=\mathscr{L}\left[q_{\varepsilon}\right]$. Since $c_{2} \leq q_{0}(x)$, we get $q(x, t) \geq c_{2}$ in $\Omega_{T}$. Therefore, $\beta(u) q(x, t)-\mu(u) \geq 0$ and by the previous arguments $u(x, t)$ is nonlocalized.

6. Final observation. As long as the functions $\beta, \mu$, and $\rho_{0}$ are relatively smooth, the system (2.5)-(2.8) will have a weak solution as defined by Eqs. (2.17), (2.18). Consider the special case $\beta(a, u)=\beta(u) b_{0} a e^{-\alpha a}$. When the birth and death moduli are constant, the population is localized if and only if $\sqrt{b_{0} \beta}<\mu+\alpha$. (When $\beta(a, u)=\beta(u) e^{-\alpha a}$, the localization condition is $\beta<\mu+\alpha[16]$.) Nevertheless, in the general nonlinear case, the birth and death functions could very well "cross" and still have localization.

As an example, note that if $\left(\sqrt{b_{0} \beta_{*}}-\alpha\right) / \beta_{*}<\mu_{*} \beta^{*}$, then the first condition in Theorem 5.1 is satisfied. If also the initial distribution is small, then the population is localized. Further, assume $\alpha \geq e \beta^{*}$; then $\sqrt{b_{0} \beta_{*}}-\alpha \leq 0$, and so the population is localized irrespective of the death modulus $\mu$. On the other hand, if $\mu^{*} / \beta_{*} \leq \sqrt{b_{0} \beta_{*}-\alpha} / \beta^{*}$, the first hypothesis in Theorem 5.3 is satisfied; so if the initial distribution is sufficiently large, the population is nonlocalized.

Figures 2-5 (see pp. 50 and 51) illustrate these cases. 


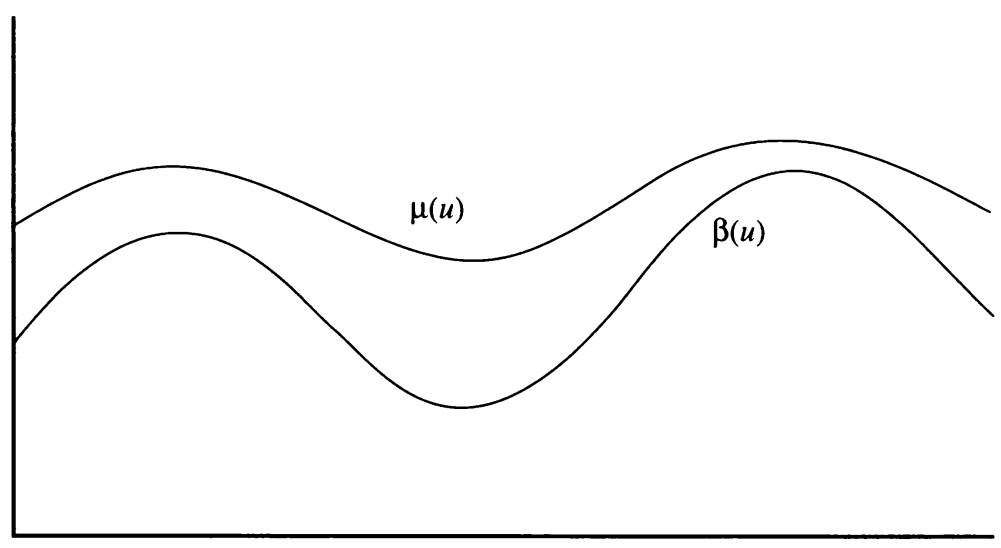

FIG. 2. $\beta(u) K_{1}<\mu(u)$ : Localization.

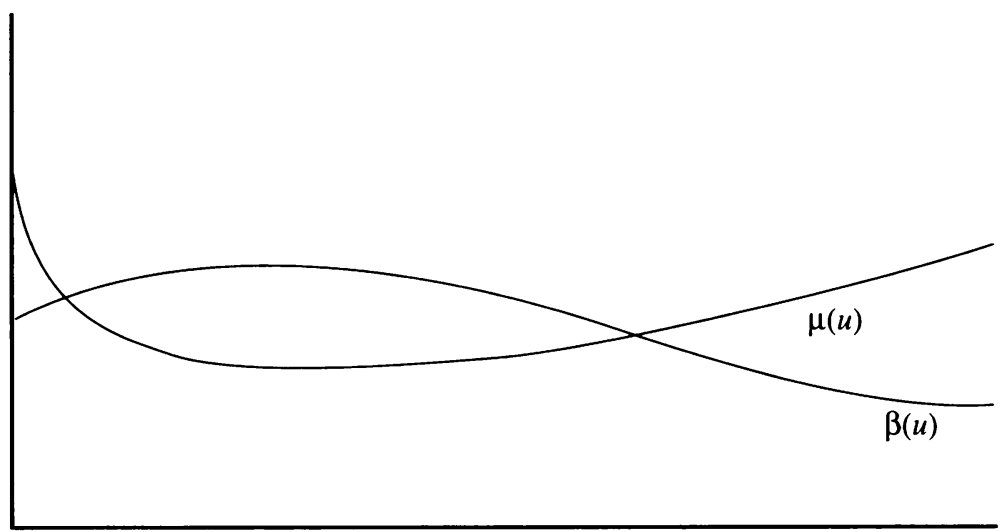

FIG. 3. $u$ is localized if the initial distribution is sufficiently small and $\left(\sqrt{b_{0} \beta^{*}}-\alpha\right) / \beta_{*}<\mu_{*} / \beta^{*}$.

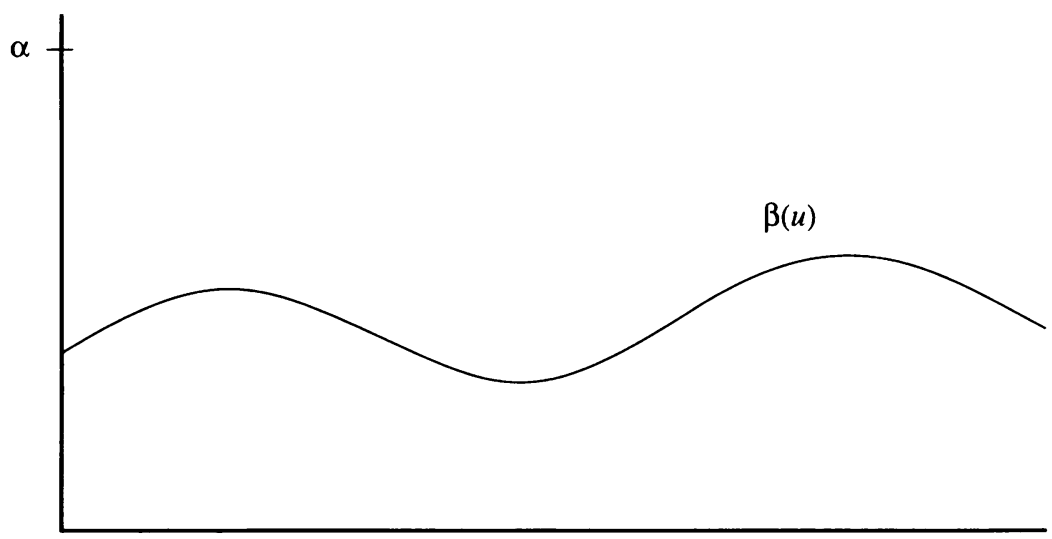

FIG. 4. If the initial distribution is sufficiently small and also $\alpha \geq$ $e \beta^{*}$, the population is localized for any $\mu$. 


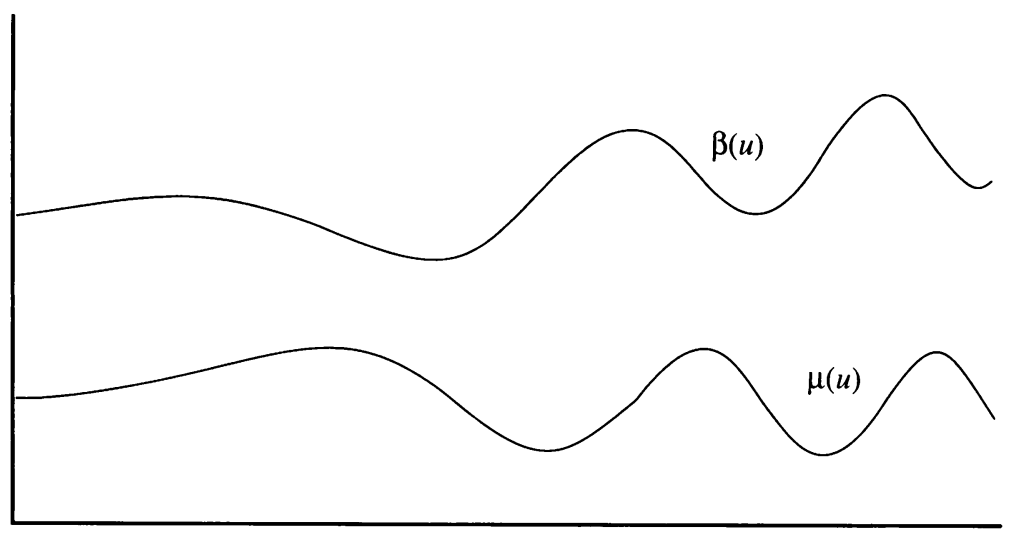

FIG. 5. If the initial distribution is sufficiently large and $\left(\sqrt{b_{0} \beta_{*}}-\alpha\right) /$ $\beta^{*} \geq \mu^{*} / \beta_{*}$, the population is nonlocalized.

\section{REFERENCES}

[1] D. G. Aronson, Regularity properties of flows through porous media, SIAM J. Appl. Math. 17, 461-467 (1969)

[2] D. G. Aronson, Regularity properties of flows through porous media: A counterexample, SIAM J. Appl. Math. 19, 299-307 (1970)

[3] Stavros Busenberg and Mimmo Ianelli, $A$ degenerate nonlinear diffusion problem in age-structured population dynamics, Nonlinear Anal. 7, 1411-1429 (1983)

[4] E. A. Carl, Population control in arctic ground squirrels, Ecology 52, 395-413 (1971)

[5] Gabriella Di Blassio, Nonlinear age dependent population diffusion, J. Math. Biol. 8, 265-284 (1979)

[6] Maria Garroni and Michel Langlais, Age dependent population diffusion with external constraints, J. Math. Biol. 14, 77-94 (1982)

[7] W. S. C. Gurney and R. M. Nibset, The regulation of inhomogeneous populations, J. Theoret. Biol. 52, 441-457 (1975)

[8] M. E. Gurtin, Some questions and open problems in continuum mechanics and population dynamics, J. Differential Equations 48, 293-312 (1983)

[9] M. E. Gurtin and R. C. MacCamy, Nonlinear age-dependent population dynamics, Arch. Rational Mech. Anal. 54, 281-300 (1974)

[10] M. E. Gurtin and R. C. MacCamy, On the diffusion of biological populations, Math. Biosci. 33, 35-49 (1977)

[11] M. E. Gurtin and R. C. MacCamy, Some simple models for nonlinear age dependence population dynamics, Math. Biosci. 43, 199-211 (1979)

[12] M. E. Gurtin and R. C. MacCamy, Diffusion models for age-structured populations, Math. Biosci. 54, 49-59 (1981)

[13] M. E. Gurtin and R. C. MacCamy, Product solutions and asymptotic behavior for age-dependent, dispersing populations, Math. Biosci. 62, 157-167 (1982)

[14] Gastón E. Hernández, Existence of solutions in a population dynamics problem, Quart. Appl. Math. 43, 509-521 (1986)

[15] Gastón E. Hernández, The Hölder property in some degenerate parabolic problems, J. Differential Equations 65, 240-249 (1986)

[16] Gastón E. Hernández, Dynamics of populations with age dependence and diffusion: Localization, Appl. Anal. 29, 143-163 (1988)

[17] Gastón E. Hernández, Anticrowding population models in several space variables, Quart. Appl. Math. 49, 87-105 (1991)

[18] A. S. Kalashnikov, On the occurrence of singularities in the solutions of the equation of nonstationary filtration, Z. Vycisl. Mat. i Mat. Fiz. 14, 891-905 (1974)

[19] Michel Langlais, A nonlinear problem in age dependent population diffusion, SIAM J. Math. Anal. 16, 510-529 (1985) 
[20] G. D. Murray, Mathematical Biology, Springer-Verlag, New York, 1989

[21] L. A. Peletier, The porous media equation, Applications of Nonlinear Analysis in the Physical Sciences (H. Amann et al., eds.), Pitman, London, 1981, pp. 229-241

[22] J. G. Skellam, Random dispersal in theoretical populations, Biometrika 38, 196-218 (1951)

[23] J. L. Vazquez, La ecuacion de los medios porosos, Actas 1 ra Jornadas de Mecanica y Fisica Matematicas, El Escorial, Sept. 1983 